\title{
The Current Clinical Practices for Prevention of Cardiovascular Disease Risk Factors in Type 2 Diabetes Mellitus
}

\author{
Aditi Garg ${ }^{1}$, Shilpa Gaidhane², Khalid Khan ${ }^{3}$, Abhay Gaidhane ${ }^{4}$ \\ 1, 2, 3Department of General Medicine, Jawaharlal Nehru Medical College, Datta Meghe Institute of Medical Sciences, \\ Sawangi, (Meghe), Wardha, Maharashtra, India. ${ }^{4}$ Department of Community Medicine, Datta Meghe Institute of \\ Medical Sciences, Sawangi, (Meghe), Wardha, Maharashtra, India.
}

\section{ABSTRACT}

\section{BACKGROUND}

India is one of the countries which has seen a rise in diabetes mellitus type 2 patient in last 2 decade. It is a known fact that patients suffering from diabetes mellitus have risk of developing many diseases like angiopathies and retionopathies. . The purpose of this study was to assess cardiovascular disease (CVD) risk factors and find out current clinical practices for prevention of cardiovascular diseases in diabetes type 2 patient attending diabetes out-patient department (OPD).

\section{METHODS}

This is a cross sectional study on 96 randomly selected patients who were diagnosed for diabetes mellitus type 2 for more than 3 months. CVD risk factors were studied using structured questionnaire about sociodemographic factors such as age, gender, family history, dyslipidaemia, hypertension, inactive physique, unsuitable food, excessive use of alcohol and all types of cigarettes; current medicines [anti-platelet medication, statins, angiotensin blocker, angiotensin converting enzyme inhibitors (ACEI)]; history of diabetes or CVD; time since diabetes is diagnosed; average number of times or frequency of follow-up and physical activity visits to health centres.

\section{RESULTS}

Present study shows abdominal obesity (84\%), unhealthy diet (86\%), physical inactivity (81\%), dyslipidaemia ( $\mathrm{n}=68,70.83 \%)$, uncontrolled diabetes $(63 \%)$, hypertension (50\%) and age more than 60 years $(41 \%)$ as the major cardiovascular diseases risk factors. Use of anti-platelet was present in $55 \%$; $74 \%$ of hypertensives were using angiotensin receptor blockers (ARBs)/ACEI; $63 \%$ of dyslipidaemia patients were using atorvastatin. This study showed that lifestyle modification has a sustained effect in preventing cardiovascular risk factors in type 2 diabetes patient.

\section{CONCLUSIONS}

Behavioural risk factors like unhealthy diet, physical inactivity, control of hypertension and hypercholesterolemia is a cause of concern in diabetes patient for preventing cardiovascular risk factor.

\section{KEY WORDS}

Type 2 Diabetes Mellitus, Clinical Practices and CVD Prevention.
Corresponding Author:

Dr. Aditi Garg,

Sharda Girls Hostel,

Jawaharlal Nehru Medical College,

Datta Meghe Institute of Medical Sciences,

Sawangi, Meghe-442001, Wardha,

Maharashtra, India.

E-mail: aditi98garg@gmail.com

DOI: $10.14260 / j e m d s / 2021 / 776$

How to Cite This Article:

Garg A, Gaidhane S, Khan K, et al. The current clinical practices for prevention of cardiovascular disease risk factors in type 2 diabetes mellitus. J Evolution Med Dent Sci 2021;10(45):3837-3842, DOI: 10.14260/jemds/2021/776

Submission 27-10-2021,

Peer Review 07-12-2021,

Acceptance 13-12-2021,

Published 21-12-2021.

Copyright (C) 2021 Aditi Garg et al. This is an open access article distributed under Creative Commons Attribution License [Attribution 4.0 International (CC BY 4.0)] 


\section{BACKGROUND}

In world, India records highest incidences of type 2 diabetes (T2DM). T2DM affects around 78 million people in India. ${ }^{1}$ Diabetic individuals have a two- to four-fold higher risk of coronary artery disease, which is equivalent to that of nondiabetic individuals who have had a previous myocardial infarction. One of the prime causes of death among diabetics is cardiovascular disease. ${ }^{2-4}$ According to studies, cardiovascular incidents occur six years earlier on an average in India than in other countries. ${ }^{5}$ Due to the comparatively younger age of commencement of cardiovascular issues, India is expected to lose 43.5 million disability adjusted life years by 2020, making coronary artery disease prevention within population at foremost risk, such as people with type 2 diabetes mellitus, a public healthcare priority.5,6

Individual cardiovascular risk factors are assessed and managed appropriately, resulting in a considerable reduction in the occurrence of cardiovascular events. When all cardiovascular risk factors are addressed in people with T2DM, macro vascular events are reduced by about $53 \%$. As a result, the most effective technique for preventing cardiovascular problems in type 2 diabetes mellitus patients is a comprehensive risk evaluation and total risk reduction therapy. ${ }^{7}$

Several studies demonstrate void in the application of screening and therapy for management of cardiovascular disease risk factors among patients with type 2 diabetes mellitus, despite proof in favour of complete interventions and fairly inexpensive medication costs to prevent cardiovascular diseases among diabetics. ${ }^{8-10}$

This is more pronounced in rural India where the accessibility of healthcare is a challenge among rural Indian population with T2DM. Hence, this study was done to determine the current clinical practices for prevention of cardiovascular disease risk factors in type 2 diabetes mellitus from rural area of central India.

\section{Aims and Objectives}

1. To count the risk factors for cardiovascular disease in people with type 2 diabetes.

2. To analyse about the present day clinical strategies for preventing cardiovascular disease risk factors in people with type 2 diabetes.

\section{METHODS}

This cross-sectional study was conducted in tertiary care rural teaching hospital of central India. Study participants were patients over 18 years of age diagnosed with type II diabetes as based on American diabetes association (ADA) criteria or are currently on glucose-lowering medication and after consent. Seriously ill patients with type 2 diabetes mellitus, and women with gestational diabetes were excluded from the study. People with type 1 diabetes were excluded.

As no population-based studies on the prevalence of CVD risk factors in people with $\mathrm{T} 2 \mathrm{DM}$ in rural India exist, we chose 50 percent prevalence of CVD as the maximum sample size at the stated absolute precision of $10 \%$ and 95 percent confidence level; the intended minimum sample size is 96 .

To analyse the presence of cardiovascular disease risk factors, participants were interviewed using a systematic questionnaire on the current practices for prevention of CVD risk factors. Information regarding demographics was collected as well. The questionnaire will be pilot tested prior to data collection.

The institutional ethics committee accepted the protocol. Participants provided written informed consent and were promised of secrecy. Participants were interviewed in their native tongue (Marathi). Data was collected for presence of cardiovascular risk factors such age, gender, family history, dyslipidaemia, hypertension, absence of physical inactivity, poor nutrition, and inappropriate use of alcohol and cigarettes in all forms; the average number of visits to a health institution and most significantly, the duration after diabetes was diagnosed, as well as current medicine. Participant's use of preventive therapy for management of CVD risk factors was based on documents or prescriptions available with participants and use of medication for prevention of CVD risk factors. Use of preventive therapy for CVDs risk factor was considered if consumed medications like anti-platelet drugs, lipid lowering drugs and blood pressure lowering drugs. Intake of medications was noted by observing rapper of empty pills and prescription of diabetes was considered. Counselling advice and appropriate management of obesity (diet and physical inactivity or medication), tobacco use and alcohol dependence were given.

A 24-hour food recall provided information regarding the diet. A mercury sphygmomanometer was used to assess blood pressure, as per JNC VII protocol.11

Weight $(\mathrm{kg})$, height $(\mathrm{m})$, waist and hip circumference (cm) were measured using World Health Organization (WHO) protocol. Waist hip ratio was calculated as per WHO protocol.12

\section{CVD Risk Factor Definitions}

Underweight - $18.5 \mathrm{~kg} / \mathrm{m} 2$, normal - $18.5-24.9 \mathrm{~kg} / \mathrm{m} 2$, overweight - $25.0-29.9 \mathrm{~kg} / \mathrm{m} 2$, and high risk $>30.0 \mathrm{~kg} / \mathrm{m} 2$ were the BMI classifications. ${ }^{13}$

A waist circumference of $>90 \mathrm{~cm}$ in men and $>80 \mathrm{~cm}$ in women, as well as a waist-to-hip circumference of 0.80 for women and 0.95 for men, was considered abdominal obesity.

Hypertension is defined as "blood pressure reading of $140 / 90 \mathrm{~mm} \mathrm{Hg}$ or higher" as per JNC VII criteria or if the patient is on antihypertensive drugs.

Cholesterol levels are determined: $<200 \mathrm{mg} / \mathrm{dl}$ high density lipoprotein (HDL) levels $<50 \mathrm{mg} / \mathrm{dl}$ in men and $<40$ $\mathrm{mg} / \mathrm{dl}$ in women, triglyceride level $<150 \mathrm{mg} / \mathrm{dl}$. Values above this were considered as uncontrolled cholesterol levels.

Nutrition risk factors were considered, if individual is consuming fruits less than twice a week and vegetables less than twice daily.

If the patient presently smokes or has previously smoked or consumed non-smoked forms of tobacco, smoking or tobacco intake was considered a risk factor.

Intake of more than one drink or $>10 \mathrm{~g}$ alcohol per day in women and two drinks or $>20 \mathrm{~g}$ alcohol per day for men and time interval between 2 drinks was regarded as negative health consequence of alcohol. 
A participant was deemed physically active if he or she did at least 150 minutes of moderate-intensity activity each week, spread out over at least three days per week with no more than two days without exercise.

The existence of a cardiovascular disease risk factor was discussed with patients by their health care provider.

\begin{tabular}{|ccc|}
\hline Sl. No. & Risk factor for CVD & Preventive Therapy \\
1 & Obesity & Dietary \& physical activities, anti-obesity drugs \\
2 & Hypertension & ACE/ARB, CCB, Diuretics \\
3 & Dyslipidaemia & Statin and any other \\
4 & Tobacco & Counselling and nicotine pouch \\
5 & Alcohol & Counselling and drugs \\
6 & Glycemic control & Hb1ac \\
\hline \multicolumn{3}{|c|}{ Preventive therapy of interest } \\
\hline
\end{tabular}

\section{Data Analysis}

Cardiovascular disease risk factors such as hypertension, dyslipidaemia, cigarette smoking, obesity, unhealthy diet, absence of physical activity, and cardiovascular disease in family were the key outcome variables. The prevalence of several risk factors in T2DM patients was calculated. The connection between risk factors and other predictors will be tested using the chi square (for categorical data) and the student's t-test (for continuous variables). The percentage of candidates who have ever had a full CVD risk assessment and are now on preventive treatment was calculated.

\section{RESULTS}

\begin{tabular}{|c|c|c|c|c|}
\hline Characteristics & Total & $\begin{array}{c}\text { Male } \\
(n=58)\end{array}$ & $\begin{array}{l}\text { Female } \\
(n=38)\end{array}$ & P Value \\
\hline Age; Mean (SD) & $58.70(9.34)$ & $59.08(9.59)$ & $58.13(9.04)$ & 0.626 \\
\hline BMI; Mean (SD) & $25.16(3.83)$ & $24.70(3.88)$ & $25.87(3.69)$ & 0.144 \\
\hline $\begin{array}{l}\text { Waist circumference; } \\
\text { Mean (SD) }\end{array}$ & $95.53(11.31)$ & $97.34(9.29)$ & $92.76(13.51)$ & $0.05(S)$ \\
\hline WHR; Mean (SD) & $0.96(0.09)$ & $0.97(0.07)$ & $0.95(0.12)$ & 0.323 \\
\hline HbA1c; Mean (SD) & $7.36(1.95)$ & $7.02(1.75)$ & $7.91(2.19)$ & $0.02(\mathrm{~S})$ \\
\hline $\begin{array}{l}\text { Fasting blood glucose; } \\
\text { Mean (SDD) }\end{array}$ & $145.52(50.72)$ & $144.75(54.06)$ & $147.13(45.81)$ & $0.341^{*}$ \\
\hline $\begin{array}{l}\text { PP blood glucose; } \\
\text { Mean (SD) }\end{array}$ & $241.08(82.44)$ & $238.71(85.03)$ & $244.71(79.29)$ & $0.729 *$ \\
\hline $\begin{array}{l}\text { Mean duration of } \\
\text { diabetes in years }\end{array}$ & $8.05(6.34)$ & $8.34(6.54)$ & $7.59(6.07)$ & $0.571(\mathrm{NS})$ \\
\hline Systolic BP; Mean (SD) & $124.89(15.07)$ & $118.44(13.48)$ & $134.73(11.79)$ & $0.000(\mathrm{~S})$ \\
\hline Diastolic BP; Mean (SD) & $78.22(9.17)$ & $74.34(9.21)$ & $82.63(7.23)$ & $0.001(S)$ \\
\hline $\begin{array}{c}\text { History of } \\
\text { Hypertension; No (\%) }\end{array}$ & $46(47.92 \%)$ & $23(39.65 \%)$ & $23(60.52 \%)$ & $0.045(S)^{* *}$ \\
\hline Triglyceride; Mean (SD) & $110.71(20.15)$ & $108.78(20)$ & $113.52(20.31)$ & $0.23^{*}$ \\
\hline Cholesterol: Mean (SD) & $177.71(24.69)$ & $172.25(24.88)$ & $184.52(23.09)$ & $0.028(\mathrm{~S})^{*}$ \\
\hline $\begin{array}{l}\text { HDL Cholesterol; } \\
\text { Mean (SD) }\end{array}$ & $39.36(3.93)$ & $39.13(3.91)$ & $39.71(3.98)$ & $0.439 *$ \\
\hline
\end{tabular}

\begin{tabular}{|c|c|c|c|c|}
\hline Characteristics & $\begin{array}{c}\text { Total } \\
\text { No }=96\end{array}$ & $\begin{array}{c}\text { Male } \\
\text { No=58 } \\
(60.42 \%)\end{array}$ & $\begin{array}{c}\text { Female } \\
\text { No=38 } \\
(39.58 \%)\end{array}$ & $\begin{array}{c}P \\
\text { Value }\end{array}$ \\
\hline \multicolumn{5}{|l|}{ Fruit serving per week } \\
\hline No & $72(75 \%)$ & $44(75.86 \%)$ & $28(73.68 \%)$ & \multirow{3}{*}{$\begin{array}{c}0.319 \\
\text { NS }\end{array}$} \\
\hline Once & $20(20.83 \%)$ & $13(22.41 \%)$ & $07(18.42 \%)$ & \\
\hline Twice or more & $04(4.16 \%)$ & $01(1.72 \%)$ & $03(7.89 \%)$ & \\
\hline \multicolumn{5}{|l|}{ Vegetables serving daily } \\
\hline No & $9(9.37 \%)$ & 7 (12.06 \%) & $2(5.26 \%)$ & \multirow{3}{*}{$\begin{array}{c}0.195 \\
\text { NS }\end{array}$} \\
\hline Once & $67(69.79 \%)$ & $42(72.41 \%)$ & $25(65.79 \%)$ & \\
\hline Twice or more & $20(20.83 \%)$ & $9(15.51 \%)$ & $11(28.94 \%)$ & \\
\hline \multicolumn{5}{|l|}{ Physical activity } \\
\hline No & $40(41.66 \%)$ & $29(50 \%)$ & $11(28.94 \%)$ & \multirow{3}{*}{$\begin{array}{c}0.11 \\
\text { NS }\end{array}$} \\
\hline Mild & $39(40.62 \%)$ & $21(36.20 \%)$ & $18(47.36 \%)$ & \\
\hline Moderate & $27(28.91 \%)$ & $8(13.79 \%)$ & $19(50 \%)$ & \\
\hline \multicolumn{5}{|l|}{ Tobacco use (any form) } \\
\hline No & $50(52.08 \%)$ & 20 (34.48 \%) & 30 (78.94 \%) & \multirow{2}{*}{$\begin{array}{c}0.000 \\
\text { (S.) }\end{array}$} \\
\hline Yes & $46(47.91 \%)$ & $38(65.51 \%)$ & $8(21.05 \%)$ & \\
\hline \multicolumn{5}{|l|}{ Alcohol * } \\
\hline No & $72(75 \%)$ & $40(68.96 \%)$ & $32(84.21 \%)$ & \multirow{4}{*}{0.205} \\
\hline Yes - Once a month & $8(9.08 \%)$ & $8(13.79 \%)$ & 0 & \\
\hline Yes - Once in week & $2(1.92 \%)$ & $2(3.44 \%)$ & 0 & \\
\hline Previous / past & $4(3.84 \%)$ & $4(6.89 \%)$ & 0 & \\
\hline
\end{tabular}

Values are No (\%); If Not Stated

Above table no 2 shows that fruit intake and vegetable consumption among the study population was very less in both males and females and merely $4.16 \%$ were consuming more than 2 servings of fruits in a week. Smoking as well as smokeless form of tobacco use was observed in $47 \%$ participants, and $25 \%$ reported use of alcohol.

Out of 96 participants, $40.62 \%$ were doing mild physical activity like household activities and $28.91 \%$ were performing 30 to 60 minutes of moderate-intensity aerobic activity daily like yoga.

\begin{tabular}{|c|c|c|c|c|}
\hline Characteristics & Total & $\begin{array}{c}\text { Male } \\
(n=58)\end{array}$ & $\begin{array}{l}\text { Female } \\
(n=38)\end{array}$ & $\begin{array}{c}P \\
\text { Value }\end{array}$ \\
\hline \multicolumn{5}{|l|}{ Age in years } \\
\hline $20-40$ & $1(1.04 \%)$ & $1(1.72 \%)$ & $0(0 \%)$ & \multirow{4}{*}{$0.48 \mathrm{NS}$} \\
\hline $41-60$ & $55(57.29 \%)$ & $31(53.44 \%)$ & $24(63.15 \%)$ & \\
\hline $61-80$ & $38(39.58 \%)$ & $28(48.27 \%)$ & $14(36.84 \%)$ & \\
\hline$>\quad 80$ & $2(2.08 \%)$ & $2(3.44 \%)$ & $0(0 \%)$ & \\
\hline \multirow[t]{2}{*}{$\begin{array}{l}\text { Waist circumference }{ }^{1} \\
\text { Abnormal }\end{array}$} & $\begin{array}{c}81 \\
(84.37 \%)\end{array}$ & $\begin{array}{c}47 \\
(81.03 \%)\end{array}$ & $34(89.47 \%)$ & \multirow{2}{*}{$0.48 \mathrm{NS}$} \\
\hline & $\begin{array}{c}15 \\
(15.62 \%)\end{array}$ & $\begin{array}{c}11 \\
(18.96 \%)\end{array}$ & $\begin{array}{c}4 \\
(10.52 \%)\end{array}$ & \\
\hline WHR (Abnormal) ${ }^{2}$ & $\begin{array}{c}76 \\
(79.16 \%)\end{array}$ & $\begin{array}{c}40 \\
(68.96 \%)\end{array}$ & $36(94.73 \%)$ & \multirow[b]{2}{*}{$0.002 \mathrm{~S}$} \\
\hline WHR (Normal) & $\begin{array}{c}20 \\
(20.83 \%)\end{array}$ & $\begin{array}{c}18 \\
(31.03 \%)\end{array}$ & $\begin{array}{c}2 \\
(5.26 \%)\end{array}$ & \\
\hline $\begin{array}{c}\text { BMI } \\
<18.5 \text { (Underweight) } \\
18.5-22.9 \text { (Normal) } \\
23-24.9 \text { (Overweight) } \\
>\quad 25 \\
\quad \text { (Obesity) }\end{array}$ & $\begin{array}{c}5(5.20 \%) \\
22(22.91 \%) \\
24(25 \%) \\
45(46.87 \%)\end{array}$ & $\begin{array}{c}5(8.62 \%) \\
15(25.86 \%) \\
12(20.68 \%) \\
26(44.82 \%)\end{array}$ & $\begin{array}{c}0(0 \%) \\
7(18.42 \%) \\
12(31.57 \%) \\
19(50 \%)\end{array}$ & $\begin{array}{l}0.212 \\
\text { NS }\end{array}$ \\
\hline $\begin{array}{l}\text { Diabetes }^{3} \\
\text { Controlled } \\
\text { Uncontrolled }\end{array}$ & $\begin{array}{l}35(36.45 \%) \\
61(63.54 \%)\end{array}$ & $\begin{array}{l}23(39.65 \%) \\
35(60.34 \%)\end{array}$ & $\begin{array}{l}12(31.57 \%) \\
26(68.42 \%)\end{array}$ & $\begin{array}{l}0.312 \\
\text { NS }\end{array}$ \\
\hline $\begin{array}{l}\text { Hypertension }{ }^{4} \\
\text { Controlled } \\
\text { Uncontrolled }\end{array}$ & $\begin{array}{l}76(79.16 \%) \\
20(20.83 \%)\end{array}$ & $\begin{array}{c}52(89.65 \%) \\
6(10.34 \%)\end{array}$ & $\begin{array}{l}24(63.15 \%) \\
14(36.84 \%)\end{array}$ & $0.002 \mathrm{~S}$ \\
\hline
\end{tabular}

1 WHR abnormal $>0.95$ in men and $>0.80$ in women

$2 \mathrm{WC}$ abnormal $>90 \mathrm{~cm}$ in men and $>80 \mathrm{~cm}$ in women

3 Diabetes controlled HbA1c $<6.5$ and uncontrolled $>/=6.5$

4 Hypertension controlled BP is SBP $<140$ AND DBP $<90$, Uncontrolled is $\mathrm{SBP}>/=140$ and $\mathrm{DBP}>/=90$

Above table describes prevalence of cardiovascular risk factors among study participants.

Obesity indicators like WC, WHR and BMI were abnormal in among $84 \%, 79 \%$ and $45 \%$ respectively. Poor glycemic control was seen in among $60 \%$ of study population. Age more than 60 years was present among $41 \%$ of study population and uncontrolled blood pressure was seen in 20 $\%$ of them. Women with higher WHR were more as compared to men (94 Vs 68) and there was high proportion of women with uncontrolled BP (36\% Vs $10 \%)$.

\begin{tabular}{|c|c|c|c|c|}
\hline Characteristics & Total & $\begin{array}{c}\text { History of } \\
\text { Hypertension }\end{array}$ & $\begin{array}{l}\text { No History of } \\
\text { Hypertension }\end{array}$ & P Value \\
\hline $\begin{array}{c}\text { Systolic BP; Mean } \\
\text { (SD) }\end{array}$ & 124.89 (15.07) & 132.17 (15.47) & 118.2 (11.19) & $0.000(\mathrm{~S})$ \\
\hline \begin{tabular}{|c} 
Diastolic BP; Mean \\
(SD)
\end{tabular} & 78.22 (9.17) & $80.21(10.43)$ & 76.4 (7.49) & $0.04(S)$ \\
\hline
\end{tabular}

Above table no 4 shows characteristics of mean blood pressure in subjects with history of hypertension. Mean systolic blood pressure (SBP) and diastolic blood pressure (DBP)of cases was 124.89 with SD 15.07 and 78.22 (SD 9.17) 
respectively. Mean systolic blood pressure (SBP) in cases having history of hypertension was 132.17 with SD 15.47 and 80.21 with SD 10.43 respectively. Mean systolic BP (SBP) and diastolic BP (DBP) in cases having no history of hypertension was 118.2 with SD 11.19 and 76.4 with SD 7.49 respectively. Systolic BP having $p$ value is 0.000 and diastolic BP having $p$ value is 0.04 .

\begin{tabular}{|c|c|c|c|c|}
\hline $\begin{array}{l}\text { Current } \\
\text { Blood } \\
\text { Pressure }\end{array}$ & $\begin{array}{c}\text { Total }(\mathrm{N}= \\
96)\end{array}$ & $\begin{array}{c}\text { History of } \\
\text { Hypertension } \\
(\mathrm{N}=46)\end{array}$ & $\begin{array}{l}\text { No History of } \\
\text { Hypertension } \\
(\mathrm{N}=50)\end{array}$ & P Value \\
\hline Control ${ }^{1}$ & $76(79.16)$ & 28 (60.89\%) & 48 (96\%) & \multirow{2}{*}{$0.000(\mathrm{~S})$} \\
\hline Uncontrolled & $20(20.83)$ & $18(39.13 \%)$ & $2(4 \%)$ & \\
\hline
\end{tabular}

1 hypertension controlled $\mathrm{BP}$ is $\mathrm{SBP}<140$ AND DBP $<90$, Uncontrolled is $\mathrm{SBP}>/=140$ and $\mathrm{DBP}>/=90$. Table 5 shows characteristics of current blood pressure levels in persons with history of hypertension. Out of 96 total cases, $79.16 \%$ have control BP less than and $20.83 \%$ have uncontrolled BP having $\mathrm{p}$ value 0.000 .

Out of 46 cases having history of hypertension, $60.89 \%$ have controlled BP and $39.13 \%$ have uncontrolled BP. Out of 50 cases having no history of hypertension, $96 \%$ have controlled BP and $4 \%$ have uncontrolled BP i.e. newly diagnosed hypertension hence total hypertensive were 48 .

\begin{tabular}{|ccccc|}
\hline HbA1C & $\begin{array}{c}\text { Total } \\
(\mathbf{N}=\mathbf{9 6})\end{array}$ & $\begin{array}{c}\text { Male } \\
(\mathbf{N}=\mathbf{5 8})\end{array}$ & $\begin{array}{c}\text { Female } \\
(\mathbf{N}=\mathbf{3 8})\end{array}$ & P Value \\
Less than 6.5 & $35(36.1 \%)$ & $23(39.65 \%)$ & $12(31.57 \%)$ & \\
6.5 to 7.4 & $25(26 \%)$ & $16(27.58 \%)$ & $9(23.68 \%)$ & 0.321 \\
\hline 7.5 to 8.4 & $15(16 \%)$ & $10(17.24 \%)$ & $5(13.15 \%)$ & \\
More than 8.4 & $21(22 \%)$ & $9(27.58 \%)$ & $12(31.57 \%)$ \\
\hline \multicolumn{5}{|c}{ Table 6. Glycemic Control } \\
\hline
\end{tabular}

Above table shows glycaemic control among study participants and its comparison between male and females. 10 subjects were having HbA1c more than or equal to 10 and among them 4 were males and 65 were females.

\begin{tabular}{|c|c|c|c|}
\hline \multicolumn{2}{|c|}{ Pharmacotherapy of DM Patients } & No & $\begin{array}{c}\text { Percent } \\
\text { (\%) }\end{array}$ \\
\hline \multirow{5}{*}{$\begin{array}{l}\text { Current anti- } \\
\text { diabetes } \\
\text { medication }\end{array}$} & Biguanides & 21 & 21.28 \\
\hline & Biguanides + Sulphonyl urea & 68 & 70.83 \\
\hline & Biguanides + Sulphonyl urea+ insulin & 3 & 3.13 \\
\hline & Biguanides + Sulphonyl urea + DPP IV Inhibitor & 4 & 4.17 \\
\hline & Other combinations of above mentioned drugs & 2 & 1.92 \\
\hline Anti-platelet & Aspirin & 53 & 55.21 \\
\hline Anti-lipid agents & Atorvastatin & 43 & 44.79 \\
\hline \multirow{5}{*}{$\begin{array}{l}\text { Antihypertensive } \\
\text { Agents }\end{array}$} & ARB & & \\
\hline & ACE Inhibitors & 32 & 69.56 \\
\hline & Calcium Chanel Blockers & 02 & 4.34 \\
\hline & Beta Blockers & 12 & 26.08 \\
\hline & Any other & 12 & 26.08 \\
\hline
\end{tabular}

Out of 96 cases, 68 (70.83\%) were on combination of biguanide and sulphonyl urea. Aspirin was taken by $55.21 \%$ cases while atorvastatin was only taken by $44.79 \%$ cases. This shows that even after having dyslipidaemia as a major risk factor, statins were taken by very less number of cases. Among anti-hypertensives, ARB is taken by $69.56 \%$ cases suggesting its good use in both hypertension prevention and cure.

\begin{tabular}{|c|c|c|c|c|}
\hline $\begin{array}{c}\text { Current Total } \\
\text { Cholesterol }\end{array}$ & $\begin{array}{l}\text { Total } \\
\text { (N 96) }\end{array}$ & $\begin{array}{l}\text { Anti-lipid } \\
\text { Drug ( } N= \\
43)\end{array}$ & $\begin{array}{c}\text { No Anti-lipid } \\
\text { Drug } \\
(\mathrm{N}=53)\end{array}$ & P Value \\
\hline Control $(<200 \mathrm{mg} / \mathrm{dl})$ & $59(61.45 \%)$ & $39(90.69 \%)$ & $20(37.73 \%)$ & \multirow[b]{2}{*}{$\begin{array}{l}0.000(\mathrm{~S}) \\
\text { chi }^{2} 12.24\end{array}$} \\
\hline $\begin{array}{l}\text { Uncontrolled } \\
(>/=200 \mathrm{mg} / \mathrm{dl})\end{array}$ & 37 (38.55 \%) & $04(09.31 \%)$ & $33(62.26 \%)$ & \\
\hline \multicolumn{5}{|l|}{ Current HDL-C } \\
\hline $\begin{array}{c}\text { Controlled } \\
\text { (<50 mg/dl men and } \\
<40 \text { in women })\end{array}$ & $\begin{array}{c}54 \\
(77.08 \%)\end{array}$ & $\begin{array}{c}36 \\
(83.72 \%)\end{array}$ & $\begin{array}{c}18 \\
(33.97 \%)\end{array}$ & \multirow{2}{*}{$\begin{array}{l}0.043(\mathrm{~S}) \\
\text { chi }^{21} 1.94\end{array}$} \\
\hline $\begin{array}{c}\text { Uncontrolled } \\
(>/=50 \mathrm{mg} / \mathrm{dl} \text { and } \\
</=40 \mathrm{in} \text { women })\end{array}$ & $\begin{array}{c}42 \\
(22.91 \%)\end{array}$ & $\begin{array}{c}7 \\
(16.27 \%)\end{array}$ & $\begin{array}{c}35 \\
(66.03 \%)\end{array}$ & \\
\hline \multicolumn{5}{|l|}{ Current Triglyceride } \\
\hline Control $(<150 \mathrm{mg} / \mathrm{dl})$ & $60(62.50 \%)$ & 39 (67.42 \%) & $21(39.62 \%)$ & \multirow{2}{*}{$\begin{array}{c}0.056 \\
\text { (NS) } \\
\text { chi }^{2} 4.238\end{array}$} \\
\hline $\begin{array}{c}\text { Uncontrolled } \\
(>/=150 \mathrm{mg} / \mathrm{dl})\end{array}$ & $36(37.5 \%)$ & $14(32.58 \%)$ & $22(60.38 \%)$ & \\
\hline
\end{tabular}

Above table shows among 96 patients, 37 (38.55 \%) have uncontrolled total cholesterol level having significant $p$ value (0.00), 42 (22.91\%) having uncontrolled HDL level having significant $p$ value $(0.043)$ and $36(37.5 \%)$ have uncontrolled triglyceride levels.

\begin{tabular}{|c|c|c|}
\hline \multirow{2}{*}{$\begin{array}{l}\text { CVD Risk } \\
\text { Factor }\end{array}$} & \multicolumn{2}{|l|}{ Intervention Received for Prevention of CVD } \\
\hline & Advice / Treatment Received & No $(\%)$ \\
\hline $\begin{array}{c}\text { Tobacco use } \\
(\mathrm{n}=46,47.91 \\
\%)\end{array}$ & Counselling for tobacco cessation & $\begin{array}{c}20 \\
(43.47)\end{array}$ \\
\hline $\begin{array}{l}\text { Harmful use of } \\
\text { alcohol } \\
(\mathrm{n}=24,25 \%)\end{array}$ & Counselling cut down harmful alcohol use & $\begin{array}{c}10 \\
(41.66)\end{array}$ \\
\hline \multirow{2}{*}{$\begin{array}{l}\text { Unhealthy diet } \\
(n=74,76 \%)\end{array}$} & Counselling for restriction of sweets in diet & $\begin{array}{c}72 \\
(97.29)\end{array}$ \\
\hline & Counselling for calories restriction and protein intake & $\begin{array}{c}72 \\
(97.29)\end{array}$ \\
\hline $\begin{array}{c}\text { Physical } \\
\text { inactivity } \\
(\mathrm{n}=79,81 \%)\end{array}$ & $\begin{array}{l}\text { Counselling for moderate to heavy physical activity }>30 \\
\text { min daily }\end{array}$ & $\begin{array}{c}74 \\
(93.67)\end{array}$ \\
\hline $\begin{array}{l}\text { BMI }(n=45 \\
46.87 \%)\end{array}$ & Counselling for weight reduction & $\begin{array}{c}30 \\
(66.66)\end{array}$ \\
\hline \multirow{2}{*}{$\begin{array}{l}\text { Blood pressure } \\
(\mathrm{n}=48,50 \%)\end{array}$} & Advice on low salt diet & $\begin{array}{c}44 \\
(91.66)\end{array}$ \\
\hline & Prescribed antihypertensive drugs & $\begin{array}{c}46 \\
(95.83)\end{array}$ \\
\hline \multirow{2}{*}{$\begin{array}{c}\text { Dyslipidaemia } \\
\text { (n=68, } 70.83 \\
\%)\end{array}$} & Advice on reducing saturated fats in diet & $\begin{array}{c}34(50 \\
\%)\end{array}$ \\
\hline & Prescribed Lipid lowering agent & $\begin{array}{c}43 \\
(63.23)\end{array}$ \\
\hline $\begin{array}{l}\text { Uncontrolled } \\
\text { diabetes } \\
(\mathrm{n}=61,63.54 \\
\%)\end{array}$ & $\begin{array}{l}\text { Adherence to medication and regular follow/up } \\
\text { (glycaemic control) }\end{array}$ & $\begin{array}{c}30 \\
(49.18)\end{array}$ \\
\hline $\begin{array}{l}\text { Anti-platelet } \\
\text { therapy } \\
(\mathrm{n}=96)\end{array}$ & & $\begin{array}{c}53(55 \\
\%)\end{array}$ \\
\hline
\end{tabular}

Out of 46 tobacco users, only 43.47 were counselled regarding tobacco cessation techniques.

Out of 25 alcohol users, only $40 \%$ were received alcohol abstinence counselling.

Even after diet counselling for weight reduction and diabetes in $97 \%$, the unhealthy diet use was seen in 74 cases. Out of 68 patients with dyslipidaemia, only 43 received atorvastatin and 34 received diet counselling. Out of 61 uncontrolled diabetes population, only 30 received counselling regarding drug adherence and importance of good glycaemic control. Out of 96 total population, only 53 received anti-platelet regularly.

\section{DISCUSSION}

\section{Baseline Characteristics of Type $2 \mathrm{DM}$}

Mean age of the patients was around 59 (9.34) years. In a multicentric ICMR-INDIA B study, mean age of type 2 
diabetes mellitus in rural population of Maharashtra was 42 years (9.34). This may be due to selection bias as ours was hospital based study.

\begin{tabular}{|ccc|}
\hline Variables in Mean & Present Study & ICMR-INDIAB \\
N & 96 & 2667 \\
Mean age (Yrs) & $58.70(9.34)$ & $42 \pm 15$ \\
Mean BMI & $25.16(3.83)$ & $20.5 \pm 3.8$ \\
Mean waist (Cms) & $95.53(11.31)$ & $72.7 \pm 11.7$ \\
Mean BP systolic (mmHg) & $124.89(15.07)$ & $127 \pm 18$ \\
Mean BP diastolic(mmHg) & $78.22(9.17)$ & $77 \pm 11$ \\
Mean HbA1c & $7.36(1.95)$ & $5.8 \pm 11$ \\
\hline Table 10. Comparison of Our Study Findings with India's First Multi- \\
centric-Epidemiological Study \\
\hline
\end{tabular}

As ours was hospital-based study, where patients seek advice at a later stage, their glycaemic control was poor, and mean duration of diabetes was more around 8 years.

\section{Prevalence of Cardiovascular Disease Risk Factors}

Our present study shows abdominal obesity (84 \%), unhealthy diet (86 \%), physical inactivity (81\%), dyslipidaemia ( $\mathrm{n}=68,70.83 \%$ ), uncontrolled diabetes $(63$ $\%$ ), hypertension (50\%) and age more than 60 years (41\%) as the major cardiovascular diseases risk factors among study participants.

In study done by A Gupta et al. among diabetes and nondiabetes, urban population shows high prevalence of key CVD risk factors like high blood pressure in $73 \%$, low high density lipoprotein cholesterol in $71 \%$, and smoke/ tobacco use of in $26 \%$ people with diabetes. ${ }^{14}$

ICMR-INDIAB community-based study in rural population shows dyslipidaemia in $77 \%$, low HDL-C in $69 \%$, physical inactivity in $50 \%$, hyper triglyceridaemia in $20 \%$, hypercholesterolemia in $13 \%$, abdominal obesity in $15 \%$ and poor glycaemic control in $50 \%$ of the population.

The greater prevalence of abdominal obesity (84\%), unhealthy diet ( $86 \%$ ), physical inactivity (81\%), in patients of diabetes is a principal finding to come across and is parallel to studies which are done in past. This may be because of elderly rural population, low education status and sparse availability of resources.

\section{Preventive Therapy Used to Prevent Cardiovascular Disease}

Use of anti-platelet was present in $55 \%, 74 \%$ of hypertensives were using ARBs/ACEI, $63 \%$ of dyslipidaemia patients were using atorvastatin.

In study done in tertiary hospitals by Sharma et al. it denotes that only 54 percent of eligible patients with coronary heart disease were recommended with a combination of four groups of medicines (aspirin, -blocker, ACE inhibitors or ARB and statins), while rates in secondary and primary care clinics were $28 \%$ and $7 \%$ respectively which is very low. 15

Our study highlights that life style risk factors are more prevalent in the study participants. There is strong evidence that lifestyle modification (regular, moderate physical activity and healthy eating habits) reduces the incidence and mortality of cardio vascular disease events in people with and without established coronary heart disease. Studies have shown that there is up to $50 \%$ and $10-20 \%$ reduction in relative risk of mortality in the general population by quitting smoking and engage in physical activity respectively employ a mixture of healthy dietary practises (low saturated fat intake, regular fish consumption, adequate fruit and vegetable intake, and low salt intake - together, a 15 - $40 \%$ reduction). Lifestyle modification is cost-effective, similar to some traditional medication therapies, and gives pre-emptive advantages ahead of metabolic abnormalities, therefore it should be used as primary intervention of choice in all diabetic patients.

The rising prevalence of cardiovascular disease in India offers a significant challenge to the country's health-care infrastructure. The application of Western countries' knowledge on CVD reduction measures, on the other hand, renders a possibility to tackle the epidemic in India. The decrease in CVD mortality in Western countries was guided by community-level changes in major risk factors and medical therapies, with improvements in community-level risk factors like cigarette use, dyslipidaemia, and hypertension that has accounted for more than half of the reduction in mortality. ${ }^{16}$

\section{CONCLUSIONS}

1. The glycemic status is poorly controlled and treated in the study.

2. The study has also shown that participants with diabetes and abdomen obesity, hypertension and dyslipidaemia are quite prevalent cardiovascular risk factors.

3. Behavioural risk factors like unhealthy diet and absence of physical activity are high among the diabetes subjects.

4. Hypertension and hypercholestolemia has been poorly controlled and is a concern in diabetic patients.

5. Prevention therapies are very poor and greatly needed for improvement in risk factor management and control in order to prevention of CVD in diabetes.

Data sharing statement provided by the authors is available with the full text of this article at jemds.com.

Financial or other competing interests: None.

Disclosure forms provided by the authors are available with the full text of this article at jemds.com.

\section{REFERENCES}

[1] International Diabetes Federation. IDF Diabetes Atlas. $6^{\text {th }}$ edn. Brussels, Belgium: International Diabetes Federation 2013.

[2] Moss SE, Klein R, Klein BE. Cause-specific mortality in a population-based study of diabetes. Am J Public Health 1991;81(9):1158-62.

[3] Geiss LS, Herman WM, Smith PJ. Mortality in noninsulin-dependent diabetes. In: National Diabetes Data Group, editor. Diabetes in America. 2nd edn. Bethesda, MD: NIH \& NIDDK: National Diabetes Information Clearing House 1995.

[4] Haffner SM, Lehto S, Ronnemaa T, et al. Mortality from coronary heart disease in subjects with type 2 diabetes and in nondiabetic subjects with and without prior 
myocardial infarction. N Engl J Med 1998;339(4):22934.

[5] Yusuf S, Hawken S, Ounpuu S, et al. Effect of potentially modifiable risk factors associated with myocardial infarction in 52 countries (the INTER HEART study): case control study. Lancet2004;364(9438):937-52.

[6] Anjana RM, Deepa M, Pradeepa R, et al. Prevalence of diabetes and prediabetes in15 States of India: results from the ICMR-INDIA B population-based crosssectional study. Lancet Diabetes Endocrinol 2017;5(8):585-96.

[7] Einarson TR, Acs A, Ludwig C, et al. Prevalence of cardiovascular disease in type 2 diabetes: a systematic literature review of scientific evidence from across the world in 2007-2017. Cardiovasc Diabetol 2018;17(1):83.

[8] Gupta R, Guptha S, Sharma KK, et al. Regional variation in cardiovascular risk factors in India: India heart watch. World J Cardiol 2012;4(4):112-20.

[9] Gupta R, Pandey RM, Misra A, et al. High prevalence and low awareness, treatment and control of hypertension in Asian Indian women. J Hum Hypertens 2012;26(10):585-93.

[10] Gupta R, Gupta A, Bhansali A, et al. High prevalence and low awareness and treatment of lipid abnormalities in urban Asian Indian subjects. Circulation 2012;125:e7089.

[11] The seventh report of Joint National Committee on prevention, detection, evaluation and treatment of high blood pressure. Bethesda (MD): National Heart, Lung, and Blood Institute (US) Report No. 04-5230, August 2004.

[12] Waist circumference and waist-hip ratio: report of a WHO expert consultation. Geneva 8-11 December, 2008.

[13] Weir CB, Jan A. BMI Classification Percentile And Cut Off Points. [Updated 2021 Jun 29]. In: Stat Pearls [Internet]. Treasure Island (FL): Stat Pearls Publishing 2021.https://www.ncbi.nlm.nih.gov/books/NBK541070

[14] Gupta A, Gupta R, Sharma KK. Prevalence of diabetes and cardiovascular risk factor in middle class urban participant in India. BMJ Open Diabetes Res Care 2014;2(1):e000048.

[15] Sharma KK, Gupta R, Agrawal A, et al. Low use of statin and other coronary secondary prevention therapies in primary and secondary care in India. Vasc Health Risk Manag 2009;5:1007-14.

[16] Prabhakaran D, Jeemon P, Roy A. Cardiovascular disease in India and current epidemiology and future directions. Circulation 2016;133(16):1605-20. 